\title{
Diagnósticos de enfermagem em portadores de hipertensão arterial na atenção primária
}

\section{Fabrizio do Amaral Mendes ${ }^{1}$, Marlucilena Pinheiro da Silva ${ }^{2}$ e Cecília Rafaela Salles Ferreira ${ }^{3}$}

\author{
1 Enfermeiro. Residente em Saúde Coletiva pela UNIFAP. Enfermeiro, Brasil. \\ E-mail: ftam1234@yahoo.com.br 1 http://lattes.cnpq.br/9964172415042851 \\ 2 Doutora em Educação, Mestre em Saúde Coletiva. Enfermeira. Docente na Universidade Federal do Amapá, Brasil.

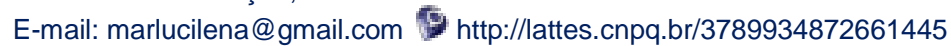 \\ 3 Enfermeira. Mestre em Ciências da Saúde pela Universidade Federal do Amapá. Enfermeira, Brasil. \\ E-mail: ceci_raphinha@hotmail.com $\mathcal{G}$ http://lattes.cnpq.br/8830057051694039
}

RESUMO: Objetivo: Identificar diagnósticos de enfermagem (DEs) em portadores de hipertensão arterial atendidos na Unidade Básica de Saúde/UNIFAP. Metodologia: Estudo descritivo de abordagem quantitativa, realizado em uma unidade básica de saúde com portadores de Hipertensão Arterial Sistêmica cadastrados e acompanhados pelo programa de Doenças Crônicas do Ministério da Saúde, no período de janeiro à maio de 2017. Resultados: 25 hipertensos passaram por consulta de enfermagem, a maioria era do sexo feminino (56\%), faixa etária que variava de 50 a 69 anos, média de idade $60,4( \pm 14)$ anos, casados (44\%), não alfabetizados (40\%) e de renda familiar de 1 a 2 salários mínimos (100\%). Os diagnósticos de enfermagem mais frequentes foram: Risco de Função Cardiovascular Prejudicada-100\%; Estilo de Vida Sedentário68\%; Falta de Adesão-60\%; Conhecimento deficiente-60\% e Sobrepeso-48\% Conclusão: A identificação dos diagnósticos de enfermagem, colabora para o avanço científico desta área e meIhora o atendimento para o usuário hipertenso, pois promove um cuidado planejado, facilitando sua implementação e favorecendo a avaliação do mesmo, contribuindo para redução da morbimortalidade advinda de complicações da HAS, provenientes da falta de adesão pelos hipertensos na Atenção Primária.

Palavras-chave: Diagnósticos de Enfermagem. Hipertensão. Atenção Primária à saúde.

\section{Nursing diagnostics in patients with arterial hypertension in primary care}

ABSTRACT: Objective: To identify nursing diagnostic (NDs) in patients with arterial hypertension treated at the Basic Health Unit / UNIFAP. Methodology: Descriptive study of a quantitative approa$\mathrm{ch}$, performed in a basic health unit with patients with systemic arterial hypertension enrolled and monitored by the Chronic Diseases Program of the Ministry of Health, from January to May 2017. Resultados: Of the 25 hypertensive patients who underwent a nursing consultation, the majority were female $(56 \%)$, ranging from 50 to 69 years, mean age $60.4( \pm 14)$ years, married $(44 \%)$, Non-literate (40\%) and family income of 1 to 2 minimum wages (100\%). The most frequent nursing diagnostic were: Risk of Impaired Cardiovascular Function-100\%; Sedentary Lifestyle-68\%; Lack of adherence-60\%; Deficiency of knowledge $-60 \%$ and Overweight- $48 \%$ Conclusion: The identification of nursing diagnostic contributes to the scientific progress of this area and improves care for the hypertensive user, since it promotes a planned care, facilitating its implementation and favoring the evaluation of the same, contributing to the reduction of morbidity and mortality due to the complications of systemic arterial hypertension due to the lack of adherence by hypertensive patients in primary care

Keywords: Nursing Diagnostics. Hypertension. Primary Health Care. 


\section{INTRODUÇÃO}

A hipertensão arterial sistêmica (HAS) é definida como uma condição clínica multifatorial caracterizada por níveis elevados e sustentados de pressão arterial (PA). Associada frequentemente a alterações das funcionalidades e/ou estruturas de órgãos-alvo, como o coração, encéfalo, rins, vasos sanguíneos e também a alterações metabólicas, relacionando a um aumento do risco de alterações cardiovasculares que podem ser não-fatais ou levar até a morte (SBC; SBH; SBN, 2010). A HAS é responsável por pelo menos $40 \%$ das mortes por acidente vascular cerebral, por $25 \%$ das mortes por doença arterial coronariana e, em combinação com o diabetes, $50 \%$ dos casos de insuficiência renal terminal (BRASIL, 2016). De acordo com a Pesquisa Nacional de Saúde realizada no Brasil em 2013, a prevalência de HAS foi de $21,4 \%$ na população de pessoas com 18 anos ou mais de idade e uma estimativa de 31 milhões de indivíduos com esta condição (BRASIL, 2015). O relatório mundial de doenças crônicas expedido pela Organização Mundial de Saúde no ano de 2014 aponta que complicações cardiovasculares como ataques do coração e acidentes vasculares encefálicos devem ser reduzidos por meio da melhoria da cobertura do tratamento farmacológico e aconselhamento de pessoas expostas a alto risco cardiovascular ou que já têm a doença, pois considera uma intervenção acessível que pode ser realizada ao nível da atenção primária, mesmo quando estes acontecem em ambientes com recursos escassos (ORGANIZAÇÃO MUNDIAL DA SAÚDE, 2014). Diante desse contexto os profissionais da Atenção Básica $(A B)$ têm um papel primordial nas estratégias de prevenção, diagnóstico, monitoriza- ção e controle da hipertensão arterial, pautando a sua práxis centrada na pessoa e, envolvendo usuários e cuidadores, em nível individual e coletivo, na definição e implementação de estratégias de monitoramento da hipertensão (BRASIL, 2013). O Enfermeiro sendo parte integrante da equipe multiprofissional deve prestar atendimento avaliando e aplicando as normatizações e direcionamentos que as instituições governamentais e profissionais propõem para benefício do seu serviço, possibilitando assim uma prática organizada que promova maior adesão ao tratamento, considerando também as demandas e contextos sociais que estes indivíduos estão inseridos. Segundo a Resolução do Conselho Federal de Enfermagem (COFEN) 358/2009 em seu art. 1 o 0 Processo de Enfermagem deve ser realizado, de modo deliberado e sistemático, em todos os ambientes, públicos ou privados, em que ocorre o cuidado profissional de Enfermagem. Sendo uma atividade privativa do enfermeiro, obedecendo a um encadeamento de cinco etapas inter-relacionadas, interdependentes e recorrentes, incluindo a identificação dos Diagnósticos de Enfermagem. A Taxonomia Internacional de Diagnósticos de Enfermagem (NANDA, 2015, p.25) conceitua:

\footnotetext{
"o diagnóstico de enfermagem como um julgamento clínico sobre uma resposta humana a condições de saúde/processos de vida, ou uma vulnerabilidade a tal resposta, de um indivíduo, uma família, um grupo ou uma comunidade."
}

Reiterando que os enfermeiros são responsáveis por diagnosticar problemas de saúde, estados de risco e disposição para a promoção da saúde. Corroborando Albuquerque; Lira; Lopes (2010) colocam que a 
identificação dos diagnósticos de enfermagem e de seus problemas colaboradores dá subsídios ao enfermeiro, para a elaboração de um plano de cuidados mais específico, de acordo com o comprometimento de cada indivíduo. E com o conhecimento de tais respostas humanas e de seus respectivos fatores preditores, torna-se possível predizer, prever, detectar e controlar complicações potenciais. Assim sendo, oferecendo um serviço sistematizado o enfermeiro, favorece a continuidade da assistência e a integração entre os diferentes membros de uma equipe multiprofissional, pois este identifica as principais demandas de saúde destes portadores de HAS e pode direcionar de uma forma mais eficaz seu tratamento ou acompanhamento na rede de saúde. Buscando prestar um atendimento integral e de qualidade ao portador de hipertensão, fez-se necessário à identificação das principais necessidades de saúde destas pessoas, reconhecendo suas particularidades biopsicossociais. Assim, esta pesquisa teve como objetivo principal, identificar os diagnósticos de enfermagem (DEs) em portadores de hipertensão arterial atendidos na UBS/UNIFAP, no período de janeiro a maio de 2017.

\section{METODOLOGIA}

Trata-se de um estudo descritivo, com abordagem quantitativa. Os dados foram coletados no período de janeiro a maio de 2017, junto aos hipertensos usuários da UBS da Universidade Federal do Amapá (UNIFAP). A referida UBS se localiza no município de Macapá no Estado do Amapá, gerenciada por convênio tripartite envolvendo a UNIFAP, a Secretaria de Saúde do Estado do Amapá (SESA) e a Secretária de Saúde do
Município de Macapá (SEMSA). As atividades de atenção primária incluem desde consultas na clínica geral, pediátrica, ginecológica, além de atendimentos de enfermagem, como consultas de enfermagem para pré-natal, preventivo do câncer de colo uterino, educação sexual e reprodutiva, educação em saúde, puericultura, controle de doenças crônicas e transmissíveis (tuberculose e hanseníase). A coleta de dados consistiu em dois momentos, em primeiro instante convidando pacientes que aguardavam na sala de espera para consulta de enfermagem voltada a pacientes com doenças crônicas, e em um segundo momento, em um retorno agendado previamente com 30 dias para uma nova consulta de enfermagem subsequente. A UBS no período da coleta de dados possuía uma população de 205 pessoas cadastradas em seu livro de atendimento do Programa de acompanhamento de doenças crônicas não transmissíveis (DCNT). Como critério de Inclusão, foi necessário ser participante assíduo do programa e não possuir outras comorbidades associadas à hipertensão, pelo fato do foco da identificação dos DEs nesta pesquisa, ser a promoção de saúde e diminuição de complicações, e além disso o aumento de demandas de saúde que possam estar relacionadas com outras doenças e não a HAS, e por fim aceitar participar do estudo, assinando o Termo de Consentimento Livre e Esclarecido da Pesquisa. Teve-se como critério de exclusão a falta em consulta subsequente, pelo fato da insuficiência de informações e dados, a não assinatura de TCLE e hipertensos com idades menores de 18 anos. Assim, obedecendo aos critérios de inclusão e exclusão, foram excluídos 88 pacientes que possuíam outras DCNT, 47 hipertensos que possuíam comorbidades as- 
sociadas como Diabetes e Cardiopatias e 5 pessoas que recusaram-se a participar da pesquisa. Obteve-se dessa forma uma amostra de hipertensos de 65 pessoas. Porém passaram por consulta de enfermagem somente 25 indivíduos com diagnóstico de HAS, sem comorbidades, de ambos os sexos, com idades entre 36 e 84 anos, pois, sendo a amostragem do tipo não probabilística por conveniência ou acessibilidade, que ocorre com frequência na prática quando o pesquisador é forçado a coletar a amostra na parte acessível da população, o que é descrito como população-amostrada, e que ocorreu nesta pesquisa, pelo fato de não se ter controle de quais hipertensos compareceriam no local da pesquisa no período da coleta, impossibilitando a utilização de outros tipos de amostragens mais confiáveis, do tipo probabilística (GIL, 2008). Para a coleta de dados, realizou-se consulta de enfermagem em consultórios da própria UBS, além de informações de fontes secundárias como os prontuários dos pacientes, e fichas de acompanhamento em doenças crônicas utilizadas pela equipe de enfermagem da UBS. Foi utilizado um instrumento de coleta de dados adaptado pelos autores da pesquisa do Caderno de Atenção Básica no 37 do Ministério da Saúde na consulta de enfermagem, composto por variáveis sociodemográficas e clínicas que subsidiaram a elaboração dos diagnósticos de enfermagem. A análise dos dados foi feita a partir do software estatístico SPSS, versão 20.0, com identificação de médias, frequências absolutas e relativas para as variáveis quantitativas, além de desvio padrão e médias. Este estudo obedeceu aos critérios de Ética em Pesquisa com seres humanos, de acordo com a Resolução do Conselho Nacional de saúde, no. 466/2012, sendo aprovado pelo
Comitê de Ética em Pesquisa da UNIFAP, parecer no 2.036.902, de 27/04/2017.

\section{RESULTADOS E DISCUSSÃO}

\subsection{Caracterização das variáveis sociode- mográficas}

A caracterização sociodemográfica desta população permite descrever o contexto social e identificar particularidades destas pessoas, incluindo variáveis como: idade, sexo, estado civil, renda familiar, ocupação e escolaridade. (Tabela 01), contribuindo assim para direcionamento no atendimento deste usuário, considerando todos os contextos que estes estão inseridos, favorecendo assim o conhecimento mais fidedigno sobre a população em estudo e permitindo a execução de atividades em saúde mais eficazes, respeitando os princípios doutrinários do sistema único de saúde.

Tabela 01. Características Sociodemográficas de Hipertensos atendidos na Unidade Básica de Saúde-UNIFAP. Macapá, AP, 2017.

Table 01. Socio-demographic Characteristics of Hypertensives attended at the Basic Health Unit-UNIFAP. Macapá, AP, 2017.

\begin{tabular}{|c|c|c|c|}
\hline Variáveis & & $\mathrm{Fn}$ & $\mathrm{F} \%$ \\
\hline \multirow{2}{*}{ Sexo } & Feminino & 14 & 56 \\
\hline & Masculino & 11 & 44 \\
\hline \multirow{10}{*}{ Ocupação } & Doméstica & 7 & 28 \\
\hline & Merendeira & 2 & 8 \\
\hline & Aposentado & 8 & 32 \\
\hline & Monitora & 1 & 4 \\
\hline & Pedreiro & 1 & 4 \\
\hline & Carpinteiro & 1 & 4 \\
\hline & Diarista & 1 & 4 \\
\hline & Copeira & 1 & 4 \\
\hline & Autônomo & 2 & 8 \\
\hline & Auxiliar Administrativo & 1 & 4 \\
\hline \multirow{3}{*}{ Idade } & $30 \mathrm{~A} 49$ & 6 & 24 \\
\hline & 50 A 69 & 11 & 44 \\
\hline & 70 A 89 & 8 & 32 \\
\hline Renda & 1 A 2 salários mínimos & 25 & 100 \\
\hline \multirow{3}{*}{ Estado Civil } & União estável & 4 & 16 \\
\hline & Casado & 11 & 44 \\
\hline & Solteiro & 9 & 36 \\
\hline
\end{tabular}




\begin{tabular}{l|l|c|c}
\hline \hline & Viúvo & 1 & 4 \\
\hline \multirow{4}{*}{ Escolaridade } & Não alfabetizado & 10 & 40 \\
\cline { 2 - 4 } & EMC* & 4 & 16 \\
\cline { 2 - 4 } & EMI* & 4 & 16 \\
\cline { 2 - 4 } & EFC* & 2 & 8 \\
\cline { 2 - 4 } & EFI* & 5 & 20 \\
\hline
\end{tabular}

*EMC: Ensino Médio Completo; EMI: Ensino Médio Incompleto; EFC: Ensino Fundamental Completo; EFI: Ensino Fundamental Incompleto.

Fonte: dados da pesquisa, 2017.

Este estudo obteve os resultados descritos a seguir. O sexo feminino foi prevalente em 56\% da amostra. Corroborando com estudo realizado por Andrade, et al. (2014), que fez a análise da prevalência de HAS autorreferida Brasileira com base nos resultados obtidos na Pesquisa Nacional de Saúde realizada pelo Instituto Brasileiro de Geografia e estatística (IBGE) no ano de 2013, apontando um percentual de 56,9\% dos entrevistados como sendo do sexo feminino. Outra pesquisa que buscou identificar a HAS autorreferida e a utilização de medicamentos de uso contínuo no Brasil, chegou ao resultado de $60 \%$ de mulheres em sua amostra (FERREIRA; BARRETO; GIATTI, 2014). Como apontam outros estudos, pode-se chegar à conclusão, que a amostra predominantemente feminina está relacionada ao fato da maior procura por assistência em saúde por este gênero, podendo assim diagnosticar a HAS em maior número nessa população (WOTTRICH et al., 2011; ANDRADE et al., 2014; DANIEL; VEIGA, 2013). 32\% dos hipertensos eram aposentados, e outros $28 \%$ da amostra referiram realizar atividades domésticas. Em estudo feito com portadores de hipertensão arterial, obteve-se resultado semelhante com aproximadamente $32 \%$ sendo aposentados e $22 \%$ executando atividades domiciliares (DANIEL; VEIGA, 2013). A média de idade foi de 60,4 anos $( \pm 14)$ e uma faixa etária preva- lente de 50 a 69 anos; observa-se uma concordância com outros estudos realizados no Brasil, com médias de idade próximas, de $64,01( \pm 9,9) ; 54,9( \pm 9,7) ; 56,7( \pm 13,1)$ respectivamente (DANIEL; VEIGA, 2013; ARAUJO; GUIMARÃES, 2007; GUIMARÃES FILHO et al., 2015 ). De acordo com metanálise realizada no Brasil, objetivando identificar a prevalência de Hipertensão em pessoas idosas, chegou-se a conclusão que $68 \%$ possuíam HAS. (PICON et al., 2013). Para as VII diretrizes Brasileiras de Hipertensão, existe uma relação direta e linear entre o envelhecer e aumento dos casos totais de HAS, associando a mudança do perfil epidemiológico atual, com mais pessoas idosas no país na última década de 2000 a 2010 (de 6,7\% para $10,8 \%)$ e consequente aumento dos anos que a pessoa vive, próximo dos 75 anos hoje em dia (SBC, 2016). A renda familiar prevaleceu com todos os entrevistados (100\%) em torno de 1 a 2 salários mínimos. Corroborando com estudos que apontam uma renda domiciliar em torno de $87,4 \%$ em hipertensos com uma renda de 1 até 3 salários mínimos no máximo (FERREIRA; BARRETO; GIATTI, 2014;). Segundo estes autores, a renda está associada a melhor adesão ao tratamento, e menciona que quanto maior a renda familiar mensal, com um ganho acima de 3 salários mínimos, menor será o número de faltas em consultas médicas nos últimos 6 meses, em contrapartida hipertensos com rendas menores que 3 salários mínimos, possuem número maior de falta em consultas médicas programadas. $\mathrm{O}$ Estado Civil que prevaleceu foi o de Casado (44\%), porém a União estável teve um percentual de $16 \%$. Em estudo realizado, obteve-se resultado $81,8 \%$ de homens que possuíam companheiras e $64,3 \%$ de mulheres que tinham companheiros, este autor asso- 
ciou a busca dos homens pelos serviços de saúde, relacionando ao fato de possuírem uma companheira, pois historicamente as mulheres buscam mais por serviços de saúde que os homens, considerando o estado conjugal destes como casados ou tendo união estável, um fator protetor para manutenção da saúde (WOTTRICH et al., 2011). Em relação à Escolaridade, este estudo identificou $40 \%$ como não alfabetizados e $28 \%$ com no máximo 8 anos de estudo; concordando com série temporal, que objetivou estimar as prevalências de HAS autorreferida na população adulta no ano de 2011 nas capitais brasileiras e no Distrito Federal, chegando ao resultado de $26,8 \%$ da amostra com até oito anos de escolaridade (ANDRADE et al., 2014). Ressalta-se nesta pesquisa, que existe uma relação inversa, entre nível de escolaridade e prevalência de HAS, sendo considerado esta variável um fator protetor contra as doenças crônicas não transmissíveis (DCNTs), pois a população com maior escolaridade acaba tendo maior acesso as práticas de promoção a saúde, como alimentação saudável, atividade física, além de alcançar mais os serviços de saúde. Reitera ainda, que a Organização Mundial de Saúde, aponta que a prevalência de HAS é maior em países de baixa e média nível educacional, porém a diminuição dos casos desta doença está diretamente relacionada às intervenções na área de saúde pública para redução de fatores de risco e aumento de fatores protetores contra a HAS.

\subsection{Caracterização dos diagnósticos de en- fermagem}

Entre os 18 diagnósticos encontrados, destaca-se o diagnóstico de Risco de Função Cardiovascular Prejudicada $(n=25)$, encon- trado em $100 \%$ dos participantes, tendo como principal Fator de Risco (FR) a presença da HAS.

Para Siqueira et al., (2015), a HAS é um importante fator de risco (FR) cardiovascular e torna-se mais preocupante quando associada a outros fatores de risco como: obesidade, sedentarismo, tabagismo, dieta inadequada, raça negra, idade avançada e baixo nível socioeconômico. Este estudo descritivo exploratório, que objetivou caracterizar o perfil de pacientes com crise hipertensiva atendidos em um pronto socorro no sul do Brasil, identificou-se que $48 \%$ das crises hipertensivas aconteceram em idosos em estágio III da HAS com idade igual ou superior a 60 anos, além de 34,6\% estarem na faixa etária de 40 a 59 anos em estágio II. Reitera que os mesmos adentram as emergências apresentando sinais clínicos de complicações cardiovasculares como: cefaleia $(71,1 \%)$, tontura $(28 \%)$, Dor precordial $(17,1 \%)$, Dor na nuca $(2,7 \%)$, entre outros sintomas, demonstrando a não adesão ao tratamento por parte destes pacientes e os riscos de complicações cardiovasculares associadas a órgãos alvo, como o Infarto Agudo do Miocárdio (IAM), Acidente Vascular Encefálico (AVE) e Insuficiência Renal Crônica (IRC).

A Obesidade (40\%), sobrepeso (48\%), Risco de sobrepeso (8\%), somados a Estilo de Vida Sedentário (68\%), foram Diagnósticos de Enfermagem que se fizeram presentes, com significativo percentual da população investigada (Tabela 02). 
Tabela 02. Diagnósticos de Enfermagem encontrados nos Hipertensos atendidos na Unidade Básica de Saúde-UNIFAP conforme NANDA 2015-2017. Macapá/AP, 2017.

Table 02. Nursing diagnoses found in hypertensive patients attending the Basic Health Unit-UNIFAP according to NANDA 2015-2017. Macapá / AP, 2017.

\begin{tabular}{|c|c|c|c|}
\hline DOMINÍO & DIAGNÓSTICO & $\mathrm{Fn}$ & $\mathrm{F} \%$ \\
\hline 01 Promoção da saúde & $\begin{array}{l}\text { Estilo de vida sedentá- } \\
\text { rio (0168) }\end{array}$ & 17 & 68 \\
\hline 01 Promoção da saúde & Falta de adesão (079) & 15 & 60 \\
\hline 02 Nutrição & Obesidade (0232) & 10 & 40 \\
\hline 02 Nutrição & Sobrepeso (0233) & 12 & 48 \\
\hline 02 Nutrição & $\begin{array}{l}\text { Risco de sobrepeso } \\
(0234)\end{array}$ & 2 & 8 \\
\hline 02 Nutrição & $\begin{array}{l}\text { Volume de líquidos } \\
\text { deficiente }(027)\end{array}$ & 1 & 4 \\
\hline 03 Eliminação e troca & Constipação (011) & 1 & 4 \\
\hline 04 Atividade/repouso & $\begin{array}{l}\text { Risco de função cardi- } \\
\text { ovascular prejudicada } \\
\text { (0239) }\end{array}$ & 25 & 100 \\
\hline 04 Atividade/repouso & Insônia (095) & 7 & 28 \\
\hline 04 Atividade/repouso & Fadiga (093) & 2 & 8 \\
\hline 05 Percepção/cognição & $\begin{array}{l}\text { Conhecimento defici- } \\
\text { ente }(0126)\end{array}$ & 15 & 60 \\
\hline 06 Autopercepção & $\begin{array}{ll}\text { Baixa } & \text { autoestima } \\
\text { situacional }(0119)\end{array}$ & 1 & 4 \\
\hline $\begin{array}{l}07 \text { Papéis e relaciona- } \\
\text { mentos }\end{array}$ & $\begin{array}{l}\text { Processos familiares } \\
\text { disfuncionais (063) }\end{array}$ & 2 & 8 \\
\hline $\begin{array}{l}09 \text { Enfrentamento/tole- } \\
\text { rância ao estresse }\end{array}$ & Ansiedade (0146) & 3 & 12 \\
\hline $\begin{array}{l}09 \text { Enfrentamento/tole- } \\
\text { rância ao estresse }\end{array}$ & Medo (0148) & 3 & 12 \\
\hline $\begin{array}{l}09 \text { Enfrentamento/tole- } \\
\text { rância ao estresse }\end{array}$ & $\begin{array}{l}\text { Sobrecarga de estresse } \\
\text { (0177) }\end{array}$ & 2 & 8 \\
\hline 11 Segurança/proteção & $\begin{array}{l}\text { Dentição prejudicada } \\
\text { (048) }\end{array}$ & 7 & 28 \\
\hline 12 Conforto & Dor crônica (0133) & 1 & 4 \\
\hline
\end{tabular}

Fonte: dados da pesquisa, 2017.

Suas respectivas $C D$ principais foram: adulto IMC> $>30 \mathrm{~kg} / \mathrm{m}^{2}$; adulto IMC> $25 \mathrm{~kg} / \mathrm{m}^{2}$; adulto com IMC aproxima-se de $25 \mathrm{~kg} / \mathrm{m}^{2}$ e Atividade Física diária inferior à recomendada para o gênero e a idade. (Tabela 03) e seus Fatores Relacionados: comportamento alimentar inadequado, comportamentos alimentares inadequados desordenados, atividade física inferior à recomendada para sexo e idade, e interesse insuficiente de apoio pela atividade física respectivamente.
Em estudo epidemiológico transversal, que objetivou analisar a distribuição espacial da obesidade em adultos em Belo horizonte identificou uma prevalência média de obesidade de $12,4 \%$ (IC=11,5-13,3) e enfatizou também que a área mais central do município possuiu uma maior aglomeração de pessoas obesas, porém sem evidência estatística. Em contrapartida, identificou também uma área de não obesos em região ao leste do município, esta com significância estatística ( $p$-valor=0,04). Estes autores associaram o número diminuído de obesos em região específica, a maior diversidade de estabelecimentos de venda de alimentos e locais para prática de atividade física, além de maior renda da vizinhança, e menores taxas de homicídio (MATOZINHOS, et al., 2015). Observa-se assim, a importância de investimento por parte dos gestores em maior infraestrutura nas cidades, direcionando recursos para incentivar a criação de locais que sejam utilizados para a prática de atividade física, além de estímulo ao comércio para venda de produtos saudáveis, como feiras e hortifrutas, objetivando o crescimento econômico e mudança de estilo de vida da população, favorecendo assim a redução da obesidade, sobrepeso e sedentarismo.

Outros diagnósticos que se destacaram devido a sua importância clínica para o correto seguimento do tratamento para a HAS, foram a Falta de adesão (60\%) e o Conhecimento deficiente (60\%) (Tabela 02). Onde tiveram destaque as CD: comportamento de falta de adesão (47\%) e conhecimento insuficiente (53\%) (Tabela 03) e Fatores Relacionados: duração prolongada do regime (67\%) e alteração na função cognitiva (60\%). 
Tabela 03. Características Definidoras dos Diagnósticos de Enfermagem elencados em hipertensos da UBS-UNIFAP conforme NANDA 2015-2017 Macapá, AP, 2017.

Table 03. Defining Characteristics of Nursing Diagnoses listed in hypertensive patients of UBS-UNIFAP according to NANDA 2015-2017 Macapá, AP, 2017.

\begin{tabular}{|c|c|c|c|}
\hline Variáveis & Característica Definidora & $\mathrm{Fn}$ & F\% \\
\hline Obesidade (0232) & Adulto $\mathrm{IMC}>30 \mathrm{~kg} / \mathrm{m}^{2}$ & 10 & 100 \\
\hline Sobrepeso (0233) & Adulto $\mathrm{IMC}>25 \mathrm{~kg} / \mathrm{m}^{2}$ & 10 & 100 \\
\hline $\begin{array}{l}\text { Estilo de vida seden- } \\
\text { tário (0168) }\end{array}$ & $\begin{array}{l}\text { AF diária inferior à } \\
\text { recomendada para o } \\
\text { gênero e a idade }\end{array}$ & 14 & 82 \\
\hline Falta de adesão (079) & $\begin{array}{l}\text { Comportamento de } \\
\text { Falta de Adesão }\end{array}$ & 7 & 47 \\
\hline $\begin{array}{l}\text { Risco de função } \\
\text { cardiovascular preju- } \\
\text { dicada (0239) }\end{array}$ & AUSENTE & 0 & 0 \\
\hline $\begin{array}{l}\text { Conhecimento defici- } \\
\text { ente (0126) }\end{array}$ & $\begin{array}{l}\text { Conhecimento insufici- } \\
\text { ente }\end{array}$ & 8 & 53 \\
\hline $\begin{array}{l}\text { Dentição prejudicada } \\
\text { (048) }\end{array}$ & Cárie nos Dentes & 7 & 100 \\
\hline Insônia (095) & $\begin{array}{l}\text { Dificuldade para manter } \\
\text { o sono }\end{array}$ & 9 & 60 \\
\hline Ansiedade (0146) & Medo & 1 & 50 \\
\hline $\begin{array}{l}\text { Processos familiares } \\
\text { disfuncionais (063) }\end{array}$ & $\begin{array}{l}\text { Deterioração nos relaci- } \\
\text { onamentos familiares }\end{array}$ & 2 & 100 \\
\hline Medo (0148) & Sensação de medo & 2 & 67 \\
\hline \multirow[t]{2}{*}{ Fadiga (093) } & Cansaço & 1 & 50 \\
\hline & $\begin{array}{l}\text { Capacidade prejudicada } \\
\text { para manter as rotinas } \\
\text { habituais }\end{array}$ & 1 & 50 \\
\hline $\begin{array}{l}\text { Baixa Autoestima } \\
\text { Situacional (0119) }\end{array}$ & $\begin{array}{l}\text { Verbalização autonega- } \\
\text { tiva }\end{array}$ & 1 & 100 \\
\hline $\begin{array}{l}\text { Sobrecarga de Estres- } \\
\text { se }(0277)\end{array}$ & Estresse excessivo & 2 & 100 \\
\hline $\begin{array}{l}\text { Risco de Sobrepeso } \\
(0234)\end{array}$ & $\begin{array}{l}\text { Adulto: IMC aproxima-se } \\
\text { de } 25 \mathrm{~kg} / \mathrm{m}^{2}\end{array}$ & 2 & 40 \\
\hline Constipação (011) & $\begin{array}{l}\text { Redução de frequência } \\
\text { nas fezes }\end{array}$ & 1 & 100 \\
\hline Dor Crônica (0133) & Expressão facial de dor & 1 & 100 \\
\hline
\end{tabular}

Fonte: dados da pesquisa,2017.

Em revisão integrativa que buscou identificar os fatores associados à não adesão ao tratamento da HAS, obteve-se o resultado que múltiplos fatores acabam predispondo os hipertensos a não aderirem ao tratamento, incluindo desde o regime terapêutico, aspectos econômicos e sociodemográficos, o serviço prestado, os profissionais de saúde inseridos na prestação dos cuidados, além de aspectos pessoais e psicossociais. Reitera que a não adesão do paciente acaba acontecendo pelo fato dos hipertensos não identificarem a sua doença como um problema de saúde que necessite de tratamento em detrimento da ausência de sintomas, enfatiza ainda, que o enfermeiro deve identificar vulnerabilidades sociais, empoderando estas pessoas, para que busquem seus direitos. Coloca ainda que quanto maior for o grau de conhecimento pelo paciente sobre a sua doença maior o envolvimento destes nas atividades de autocuidado e adesão ao tratamento, com responsabilidade de toda equipe de saúde para promoção e implementação de estratégias educacionais que orientem e eduquem estes hipertensos (SILVA, et al., 2016).

Os entrevistados apresentaram também, diagnósticos de enfermagem diretamente ligados ao emocional e a saúde mental, como ansiedade (12\%), insônia (28\%), medo $(12 \%)$, sobrecarga de estresse (8\%) e baixa autoestima situacional (4\%), Outros diagnósticos ainda, podem também ser observados na Tabela 02.

Como coloca Lipp (2007), o tratamento da HAS, representa um grande desafio para os profissionais da saúde, pois, mesmo com todos os avanços no tratamento medicamentosos da doença, a equipe de saúde acaba vendo-se frustrada ao verificar a resistência por parte destes pacientes à mudança de estilo de vida, tão essencial para uma terapêutica eficaz. Enfatiza que os profissionais seu atendimento na doença do paciente e não no paciente, não identificando este como um ser humano, que possui desejos, fantasias, medos e disposições cognitivas, e que nem sempre irão acabar reagindo de modo colaborativo. Aponta também que é necessário conhecer o portador de HAS, para saber o que pode motivar e o que pode interferir no processo do 
autocuidado, pois o mesmo tende a usar mecanismos de defesa, como a negação, que podem contribuir para a não adesão ao tratamento. Este estudo chegou a conclusão que o Treino de Controle de stress (TCS) é eficaz na modulação da reatividade cardiovascular em momentos de stress emocional e que auxilia tanto na adesão ao tratamento, quanto na reestruturação do modo de pensar, conduzindo a uma mudança estável e duradoura do estilo de vida, mais compatível com o autocuidado necessário no manejo de doenças crônicas. Percebe-se assim, que alterações emocionais, como, a baixa autoestima, ansiedade, medo e principalmente o estresse, podem ser fatores dificultantes no processo de adesão do hipertenso, e prejudiciais para aumento do risco cardiovascular, facilitando assim o surgimento de emergências hipertensivas e lesões em órgãos alvo. Os DE elencados podem ser visualizados na Tabela 02, assim como suas características definidoras na Tabela 03 e Fatores relacionados na Tabela 04.

Tabela 04. Fatores Relacionados e Fatores de Risco Prevalentes dos Diagnósticos de Enfermagem. Macapá, AP 2017.

Table 04. Related Factors and Prevalent Risk Factors of Nursing Diagnoses. Macapá, AP 2017.

\begin{tabular}{llcc}
\hline Variáveis & Fatores Relacionados & Fn & F\% \\
\hline Obesidade (0232) & $\begin{array}{l}\text { Comportamento alimen- } \\
\text { tar inadequado }\end{array}$ & 8 & 47 \\
\hline Sobrepeso (0233) & $\begin{array}{l}\text { Comportamentos ali- } \\
\text { mentares inadequados } \\
\text { desordenados }\end{array}$ & 11 & 92 \\
\hline $\begin{array}{l}\text { Estilo de vida se- } \\
\text { dentário (0168) }\end{array}$ & $\begin{array}{l}\text { Interesse insuficiente de } \\
\text { apoio pela atividade } \\
\text { física }\end{array}$ & 10 & 59 \\
\hline $\begin{array}{l}\text { Falta de adesão } \\
\text { (079) }\end{array}$ & $\begin{array}{l}\text { Duração prolongada do } \\
\text { regime }\end{array}$ & 10 & 67 \\
\hline $\begin{array}{l}\text { Risco de função } \\
\text { cardiovascular } \\
\text { prejudicada (00239) }\end{array}$ & HAS & 25 & 100 \\
\hline $\begin{array}{l}\text { Conhecimento } \\
\text { deficiente (0126) }\end{array}$ & $\begin{array}{l}\text { Alteração na função } \\
\text { cognitiva }\end{array}$ & 9 & 60 \\
\hline $\begin{array}{l}\text { Dentição prejudica- } \\
\text { da (048) }\end{array}$ & $\begin{array}{l}\text { Dificuldade de acesso a } \\
\text { cuidados dentários }\end{array}$ & 7 & 50 \\
\hline \hline
\end{tabular}

\begin{tabular}{|c|c|c|c|}
\hline & profissionais & & \\
\hline Insônia (095) & Desconforto físico & 9 & 60 \\
\hline \multirow{4}{*}{ Ansiedade (0146) } & Preocupação & 1 & 33,3 \\
\hline & Ameaça de morte & 1 & 33,3 \\
\hline & Crise situacional & 1 & 33,3 \\
\hline & $\begin{array}{l}\text { Necessidades não aco- } \\
\text { Ihidas }\end{array}$ & 1 & 33,3 \\
\hline $\begin{array}{l}\text { Processos familiares } \\
\text { disfuncionais (063) }\end{array}$ & Abuso de Substância & 2 & 100 \\
\hline Medo (0148) & Estimulo fóbico & 3 & 100 \\
\hline \multirow[t]{3}{*}{ Fadiga (093) } & Condição fisiológica & 1 & 33,3 \\
\hline & $\begin{array}{l}\text { Falta de condicionamen- } \\
\text { to físico }\end{array}$ & 1 & 33,3 \\
\hline & Privação de sono & 1 & 33,3 \\
\hline $\begin{array}{l}\text { Baixa autoestima } \\
(0119)\end{array}$ & Historia de perda & 1 & 100 \\
\hline $\begin{array}{ll}\text { Sobrecarga } & \text { de } \\
\text { estresse (0177) } & \\
\end{array}$ & Recursos insuficientes & 2 & 67 \\
\hline $\begin{array}{l}\text { Risco de sobrepeso } \\
(0234)\end{array}$ & $\begin{array}{l}\text { AF diária inferior a reco- } \\
\text { mendada para sexo e } \\
\text { idade }\end{array}$ & 2 & 40 \\
\hline $\begin{array}{l}\text { Volume de líquidos } \\
\text { deficiente (027) }\end{array}$ & $\begin{array}{l}\text { Alteração no turgor da } \\
\text { pele }\end{array}$ & 1 & 100 \\
\hline Constipação (011) & $\begin{array}{l}\text { AF Media diária inferior } \\
\text { à recomendada pra } \\
\text { idade e o gênero }\end{array}$ & 1 & 100 \\
\hline Dor crônica (0133) & $\begin{array}{l}\text { Condição musculoes- } \\
\text { quelética crônica }\end{array}$ & 1 & 100 \\
\hline $\begin{array}{l}\text { Volume de liquido } \\
\text { inadequado (027) }\end{array}$ & $\begin{array}{l}\text { Alteração no turgor da } \\
\text { pele }\end{array}$ & 1 & 100 \\
\hline
\end{tabular}

$\mathrm{AF}^{*}$ : Atividade Física.

Fonte: dados da pesquisa, 2017.

\section{CONSIDERAÇÕES FINAIS}

A Hipertensão Arterial Sistêmica (HAS) vem sendo considerada por diversos pesquisadores como um agravante a saúde da população, apresentando prevalência assustadora a nível nacional e mundial, além de ser causadora de índices elevados de morbidade e mortalidade.

A atenção primária de saúde tem como uma de suas principais funções a identificação precoce de diagnóstico de HAS e estimulo a promoção e prevenção deste agravo. Além de identificar fatores de risco que podem contribuir para o desenvolvimento desta patologia, prestando assistência de qualidade e resolutiva com atendimento de 
equipe multiprofissional articulada para resolução das necessidades de saúde na atenção primária.

O Enfermeiro neste contexto possui papel extremamente importante, pois seu atendimento na maioria dos casos é o inicial, e baseia-se na busca para identificar as problemáticas e anseios do paciente e direcionar os atendimentos de saúde. Além disso, o papel do enfermeiro através da consulta de enfermagem permite um atendimento mais particularizado, sistematizado e com um olhar integral.

A identificação dos diagnósticos de enfermagem (Tabela 01) colabora para o avanço científico da área de enfermagem, pois por meio da identificação dos mesmos, o atendimento para o paciente hipertenso torna-se planejado, facilita sua execução e favorece a avaliação do cuidado, além de considerar as reais necessidades do usuário, contribuindo para identificação de demandas que muitas vezes não são detectadas sem o desenvolvimento de uma prática mais holística e humanizada.

Este estudo identificou como principais necessidades de saúde, a falta de adesão no tratamento, conhecimento deficiente, obesidade e sobrepeso, sedentarismo, risco de função cardiovascular e outras demandas relacionadas ao contexto psicossocial do indivíduo, como ansiedade, estresse, medo, baixa autoestima e processos familiares disfuncionais, que podem ser minimizadas e/ou solucionadas se o processo de enfermagem acontecer nesses ambientes de atenção primária, focando em um atendimento humanizado, interdisciplinar e resolutivo, contribuindo para a redução dos casos alarmantes de mortalidade advindas de complicações desta patologia.

\section{REFERÊNCIAS}

ALBUQUERQUE, J. G.; LIRA, A. L. B. C.; LOPES, M. V. O. Fatores preditivos de diagnósticos de enfermagem em pacientes submetidos ao transplante renal. Rev. Bras. Enferm., Brasília, v.63, n.1, p. 98-103, 2010.

ANDRADE, S. S. C. A. et al. Prevalência da hipertensão arterial autorreferida nas capitais brasileiras em 2011 e análise de sua tendência no período de 2006 a 2011. Rev Bras Epidemiol Suppl Pense, Brasília, p.21526, 2014.

ARAUjo, J. C.; GUIMARÃES, A. C. Controle da hipertensão arterial em uma unidade de saúde da família, Rev Saúde Pública, Salvador, v. 41, n.3, p.368-74, 2007. https://doi. org/10.1590/S0034-89102007000300007 BRASIL. Ministério da saúde. Portal da Saúde, 2016. Disponível em: <http://portalsau de.saude.gov.br/index.php/o-ministerio/pri ncipal/secretarias/814-sasraiz/daet-raiz/do encas-cronica//1-doencas-cronica/22067-hi pertensao-arterial-e-diabetes $>$. Acesso em: 01 maio 2017.

- Ministério da Saúde. Cadernos de Atenção Básica, n.37. Secretaria de Atenção à Saúde. Departamento de Atenção Básica. Brasília: Ministério da Saúde, 2013. 128 p. il. - Ministério da Saúde. Secretaria de Vigilância em Saúde. Saúde Brasil 2014: uma análise da situação de saúde e das causas externas. Brasília: Ministério da Saúde, 2015. 462 p. il.

CONSELHO FEDERAL DE ENFERMAGEM. Resolução COFEN-358/2009. Dispõe sobre a Sistematização da Assistência de Enfermagem e a implementação do Processo de Enfermagem em ambientes, públicos ou privados, em que ocorre o cuidado profissional de Enfermagem, e dá outras providências. Disponível em: <http://www.cofen.gov.br/ 
resoluo-cofen-3582009_4384.html> Acesso em: 20 mar. 2016.

DANIEL, A. C. Q. G., VEIGA, E. V. Fatores que interferem na adesão terapêutica medicamentosa em hipertensos. Einstein, São Paulo, v.11, n.3, p.331-7, 2013. https://doi.org/ 10.1590/S1679-45082013000300012

FERREIRA, R. A.; BARRETO, S. M.; GIATTI, L. Hipertensão arterial referida e utilização de medicamentos de uso contínuo no Brasil: um estudo de base populacional. Cad. Saúde Pública, Rio de janeiro, v.30, n.4, p.81526, abr. 2014.

GIL, A. C. Métodos e técnicas de pesquisa social. 6. ed. São Paulo: Atlas, 2008.

GUIMARÃES FILHO, G. C. et al. Evolução da Pressão Arterial e Desfechos Cardiovasculares de hipertensos em um Centro de Referência. Arq Bras Cardiol, Goiás, v. 41, n.3, p.292-98, 2015.

LIPP, M. E. N. Controle do estresse e hipertensão arterial sistêmica. Rev Bras Hipertens, Campinas, v.14, n.2, p.89-93, 2007.

MATOZINHOS, F. P. et al. Distribuição espacial da obesidade em área urbana no Brasil. Ciência \& Saúde Coletiva, Belo Horizonte, v. 20, n. 9, p. 2779-86, 2015. https://doi.org/ 10.1590/1413-81232015209.00442015 NANDA. International, Diagnósticos de Enfermagem da NANDA: definições e classificação 2015-2017, Porto Alegre: Artmed, 2015.

ORGANIZAÇÃO MUNIDAL DA SAÚDE. Relatório Mundial sobre as doenças não transmissíveis. 2014. Disponível em <http://app s.who.int/iris/bitstream/10665/148114/1/9 789241564854_eng.pdf?ua=1 $>$ Acesso em 14 fev. 2016.

PICON, R. V. et al. Prevalência de Hipertensão entre Pessoas Idosas no Brasil Urbano: Revisão Sistemática com Meta-Análise. Am J Hypertens, v.26, n.4, p.541-548, 2013. htt ps://doi.org/10.1093/ajh/hps076

SILVA, A. P. Fatores associados à não adesão ao tratamento da hipertensão arterial sistêmica: uma revisão integrativa. J: res.:care online, Piauí, v.8, n.1, p.4047-55, jan./mar., 2016.

SIQUEIRA, D. S. et al. Perfil de pacientes com crise hipertensiva atendidos em um pronto socorro no sul do Brasil. Rev Enferm UFSM. Porto Alegre, v.5, n.2, p.224-234, abr./jun., 2015.

SOCIEDADE BRASILEIRA DE CARDIOLOGIA. VII Diretrizes Brasileiras de Hipertensão. Arq Bras Cardiol. v.107, n.3, setembro 2016. WOTTRICH, S. H. et al. Gênero e manifestação de stress em hipertensos. Estudos de Psicologia, Campinas, v.28, n.1, p. 27-34, jan./ mar., 2011.

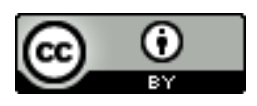
access article distributed under the terms of the Creative Commons Attribution License, which permits unrestricted use, distribution, and reproduction in any medium, provided the original work is properly cited.

Artigo recebido em 04 de agosto de 2017.

Avaliado em 15 de dezembro de 2017.

Aceito em 22 de janeiro de 2018.

Publicado em 25 de maio de 2018.

\section{Como citar este artigo (ABNT):}

MENDES, Fabrizio do Amaral; SILVA, Marlucilena Pinheiro da; FERREIRA, Cecília Rafaela Salles. Diagnósticos de enfermagem em portadores de hipertensão arterial na atenção primária. Estação Científica (UNIFAP), Macapá, v. 8, n. 1, p. 91-101, jan./abr. 2018. 